\title{
Assessing absolute and relative accuracy of recreation grade and mobile phone GNSS devices: a method for informing device choice
}

Martin Schaefer and Tara Woodyer

\begin{abstract}
The affordability/availability and portability of recreation-grade receivers and mobile devices, compared with commercial survey Global Navigation Satellite Systems (GNSS), commonly referred to as Global Positioning System, make them worthy of consideration for student fieldwork and research. While empirical evidence of the relative accuracy of low-cost GNSS receivers in open areas is currently limited, initial findings suggest that levels of relative accuracy, as opposed to absolute accuracy, make these devices an inexpensive and manageable technology for measuring the relative location of land features. Given the current speed at which GNSS technology advances, it is important to have an appreciation of any proposed device's accuracy. This paper presents a method for evaluating the levels of absolute and relative accuracy of various devices, which can inform the choice of device for particular survey-based projects. It can also be used to indicate a 'best practice' approach to using GNSS devices in a research and study context, through an assessment of the factors influencing levels of relative accuracy, such as the length of time a device is left to settle before recording a point. In addition, this method can be a useful exercise in and of itself for helping students better understand absolute and relative accuracy, the capabilities of their own mobile hardware, and the potential use of recreation-grade and mobile devices in a range of scenarios both within and beyond the academy.
\end{abstract}




\section{Introduction}

In geographical research and teaching the spatial interaction between features is often central to the research question. There are a number of surveying techniques that can be employed to locate a feature in absolute terms (absolute accuracy), as in where on earth it is, or in relative terms (relative accuracy), as in where a feature is located in relation to other features. The technique employed has to be determined by the required scale of the project and available equipment.

The accuracies in both terms can vary from sub-centimetre to $15 \mathrm{~m}$ and the cost from USD 50 to tens of thousands of dollars. This study focuses on the use of recreation grade and mobile phone GPS receivers and their respective accuracies. The former are inexpensive whereas the latter are now ubiquitous in modern smart phones. It is the affordability/availability and portability that make these devices so useful to student fieldwork and research. Increasingly they are being used not only in more traditional surveying applications, but also in broader applications such as the mapping of emotion (Al-Husain et al 2013).

Recreation grade GPS receivers cost around GBP 60 or USD 50 (Search of UK and US online retailers April 2014), compared to GBP 20-30,000 for commercial survey devices, and provide a stated horizontal accuracy of $3-6 \mathrm{~m}$. This is based on the figures for absolute accuracy stated by the devices used in this study. In practical use it has often been found that the relative accuracy of recreation grade GPS receivers used in a survey over a short time window is better than the stated absolute accuracy, for example, a survey carried out in the space of a morning versus over the space of a few days. The theoretical understanding of 
GPS supports this, as the GPS error is mainly systematic in nature and consistent over short periods of time and in small areas. GPS accuracy is not normally given by the manufacturers of GPS enabled mobile phones; however, their ubiquity makes them worthy of attention. A search on GSMArena.com phone finder tool (August 2014) reveals that $63 \%$ of phones and $85 \%$ of touchscreen phones released from 2010 onwards are GPS enabled.

In this paper a method is presented for assessing the absolute and relative accuracy of a device, recreation grade or mobile, to inform device choice for fieldwork or research projects. Particular attention is paid to whether relative accuracy can be regarded as higher than the stated absolute accuracy, and the factors influencing relative accuracy of recreation grade GPS receivers and mobile phone devices. If the relative accuracy of these devices is quantified both professional and student researchers could broaden the scope of projects that these inexpensive systems can be used in if absolute accuracy is not the primary research outcome.

Following an introduction to GPS errors, accuracy and survey alternatives, this paper outlines a method for evaluating the level of accuracy of recreation grade receivers and mobile phone devices. This is based on two case studies conducted at different sites in the south of England. The implications of the accuracy findings from these case studies are then discussed in relation to practical field survey techniques to demonstrate the potential use of the method for informing device choice. 


\section{GPS errors and accuracy}

A Global Navigation Satellite System (GNSS) utilises a constellation of satellites emitting microwave signal for location and timing purposes. GPS is the common term used, although in strict terms it only applies to the American Navstar system. Other GNSS exist (GLONASS) or are in active development (Gallileo, BeiDou) (Gleason and Gebre-Egziabher 2009; Kaplan and Hegarty 2006). Recreation grade receivers and mobile phones are increasingly using both GPS and GLONASS to provide complement each other for position fixing (Andersen et al 2009; GSMArena.com 2014; O’Shea 2013; OXTS 2014).

In broad terms a GNSS satellite sends out carrier waves embedded with two types of information. The first is the navigation message and the second is a Time of Arrival (TOA) signal that determines the distance between the satellite and the receiver. Four satellites are required to obtain a fix in three dimensions (Kaplan and Hegarty 2006; van Sickle 2008). The major components of the navigation signal are the GNSS time, a clock correction signal, the ephemeris of the satellite and atmospheric correction (van Sickle 2008). The TOA signal tells the receiver how long the signal took to travel to the receiver from the control point, i.e the satellite (Kaplan and Hegarty 2006).

There are four main factors influencing the uncertainty of a GNSS position: a time bias; an ephemeris bias; a receiver bias; and atmospheric and constellation bias. These are addressed to some extent by the data in the navigation signal, which are uploaded daily; however, accuracy continuously deteriorates following an upload (Kaplan and Hegarty 2006).

The time bias results from the TOA signal supposing that the clocks in the satellite and the receiver are both accurate and synchronous. The satellites carry up to 4 atomic clocks, which are both stable and accurate (van Sickle 2008). They do, however, still drift and have to be 
corrected from ground based control stations (van Sickle 2008; Kaplan and Hegarty 2006). This correction is passed on from the satellite to the receiver using the navigation signal, which is why GNSS signals are not only used for navigation purposes, but also for timesensitive transactions, such as scientific measurements or financial transactions. There will still, however, be a discrepancy between the atomic clocks of the satellites and the crystal based clocks in the recreation grade receivers. There is also a correction for ambiguity due to general and special relativity (van Sickle 2008; Kaplan and Hegarty 2006).

The ephemeris bias results from the assumption that the satellite is a precise control point based on the current ephemeris data. The ephemeris, however, is a predicted position that is again uploaded by the control station based on its observations of the satellite's orbit; the actual position might vary (Kaplan and Hegarty 2006).

The atmospheric bias is due to attenuation of the TOA signal used for finding the distance to the satellite by mainly ionospheric and also atmospheric conditions. Once the signal hits the earth's ionosphere and atmosphere it is attenuated, initially slowed down, but it can also be sped up again by various factors. The atmospheric conditions are modelled and part of the navigation signal; an additional problem, however, is that, depending on the satellite's position in the sky relative to the receiver, the signal can travel a short or a long distance through the atmosphere. "Three times as much delay is incurred when viewing satellites at low elevations than at the zenith" (Kaplan and Hegarty 2006, 314).

It follows that the constellation of the satellites has an effect on the accuracy of the timing signal. In addition to the elevation controlling the time of travel of the signal through the atmosphere, the relative position of the satellites also influences the geometry of the area of locational uncertainty, the dilution of precision (DOP) as illustrated in figure 1 (Kaplan and 
Hegarty 2006). In addition to these stated biases, a small variation can be introduced by the receiver hardware (van Sickle 2008).

Figure 1: Positional dilution of precision (based on Kaplan and Hegarty 2006, 322) a) Low DOP b) High DOP

a)

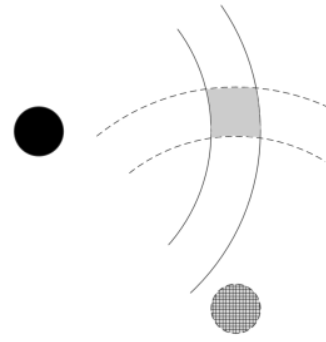

GPS 1
GPS 2 b)
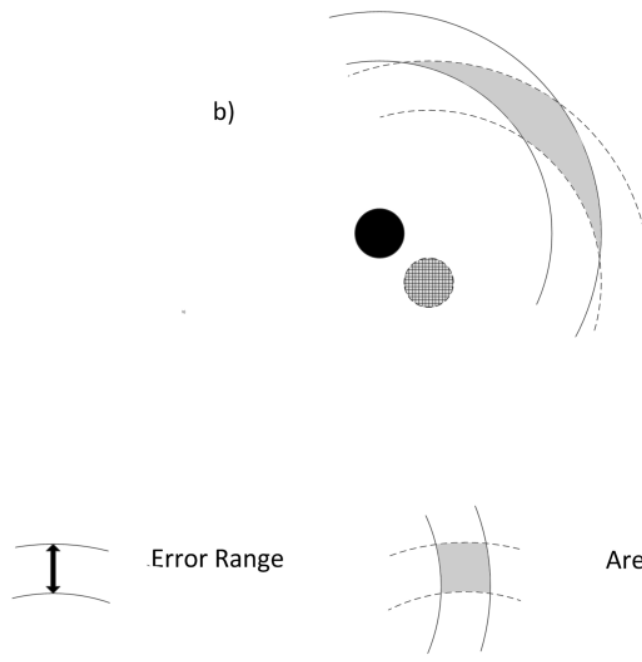

Area of potential position

Finally, the immediate environment can have a big impact on the receiver bias through multipath error. The signal from a GNSS satellite can, in addition to the straight line path, arrive at the receiver by multiple paths by reflecting off nearby objects and the ground (Kaplan and Hegarty 2006; Franchois 2005; Townsend and Fenton 1994; van Sickle 2008). This can be a major source of error (Franchois 2005).

These factors combine to produce the stated locational uncertainty of 3-6 metres of recreation grade receivers. The above uncertainty only addresses systematic error sources and assumes a clear view of the sky. In forested areas foliage has a major impact on GNSS accuracy (Andersen et al. 2009; Rodríguez-Pérez et al. 2007; Sigrist et al. 1999). 
To address these issues several geostationary Satellite Based augmentation systems (SBAS) have been deployed to ensure GNSS meet the requirements of aviation authorities for positioning. SBAS, such as the US-American WAAS, the European EGNOS, Indian GAGAN or the Japanese MSAS, provide ionosphere, orbit and clock corrections in real time over a wide area. They can provide an accuracy of $1-2 \mathrm{~m}$ in ideal conditions (Andersen et al 2009; Heßelbarth and Wanninger 2012; Jyh-Ching et al 2013)

Whilst all these factors affect the absolute accuracy of a survey, they are, however, systematic in nature, consistent over a location and change only slowly over short periods of time. Within a given location and timeframe the effect on the relative accuracy should be less. If this is correct, recreation grade receivers could be used in studies that require a higher degree of relative accuracy than the stated absolute GNSS accuracy.

\section{Survey alternatives and accuracy}

For accurate locating of a feature a differential GNSS (DGNSS) can be employed. Such a system uses two receivers to remove the systematic error inherent to GNSS (Longley et al. 2011). A commercial survey DGNSS usually consists of two receivers, with one set up on a known location and broadcasting a correction signal to a roving unit. The correction signal can come from a broadcast source as well, if available and supported by the system. In the UK, the Ordnance Survey (OS) provide a facility for downloading correction information for post processing of data after a survey (OS 2013). A survey DGNSS system can achieve centimetre accuracy. 
For relative location of features a Total Station (TS) can be utilised. A TS measures angles and distances from a known point, given an initial orientation. If the known point is an accurate point derived from a DGNSS or a benchmark and the initial orientation can be set to an equally accurate point this can give high degrees of both absolute and relative accuracy. The relative accuracy of such a system can be in the sub-centimetre range (Uren and Price 2006).

The complexity of these systems comes at a financial and practical cost in terms of research projects and student teaching. Even if these expensive systems are present in a research or teaching environment the deployment might not be practical; the whole instrument setup is difficult to transport without vehicular support and expert support and/or training are needed to set up and use the system. In research terms these factors can be accounted for in any costing or grant proposal. In teaching terms it is difficult to support individual projects with this resource. When air travel is involved additional costs are usually incurred. The value and complexity of the kit requires supervision of students in the field, adding staff costs. These costs mean that few of these instruments are available and the pressure on such a valuable resource can be high. Recreation grade receivers and mobile phones provide a less expensive alternative, if they satisfy the absolute or relative accuracy requirements of a given study.

In the following section we set out a method for evaluating the level of relative accuracy of recreation grade receivers and mobile phone devices, which can be used to inform device choice for research and teaching projects. 


\section{A Method for Evaluating Relative Accuracy Levels of recreation grade receivers and GPS enabled mobile phone devices}

To evaluate the respective levels of accuracy of recreation grade receivers and mobile phone devices, two case studies were set up in separate locations in the south of England. Either a DGNSS or a combination of DGNSS and TS was used to obtain the accurate coordinates of easily recognisable features. These features were then measured using a variety of test devices. The first case study concentrated on recreation grade receivers, whereas the second study was conducted with student volunteers and their mobile phones (Table I).

Table I: Summary of devices

Case Study 1: Sinah Gun site, Hayling Island

\begin{tabular}{|l|l|}
\hline Device & Points collected \\
\hline Garmin & 130 \\
\hline Magellan & 155 \\
\hline Total & $\mathbf{2 8 5}$ \\
\hline
\end{tabular}

Case Study 2: Southsea Promenade

\begin{tabular}{|l|l|}
\hline Device & Points Collected \\
\hline Apple 4, 5, 5c & 340 \\
\hline Samsung S3mini, S4 & 120 \\
\hline Sony Xperia E, P, Z & 158 \\
\hline Total & $\mathbf{6 1 8}$ \\
\hline
\end{tabular}

The method of evaluation involved assessing both the absolute and relative accuracy of the various test devices. To assess the absolute accuracy, the distance between each accurate point and the respective sample point is calculated along with the azimuth of the error, e.g. point $a$ is measured by a recreation grade receiver to be $3.5 \mathrm{~m}$ from true $a$ at an azimuth of 45 degrees, i.e. $3.5 \mathrm{~m}$ off to the north-east. To assess the relative accuracy of the various devices, the control data is used to calculate the correct distance and azimuth between each point in the control data set. For each test data set the same calculations are performed and compared to the accurate data, e.g. if point $a$ and $b$ are $5 \mathrm{~m}$ apart at an azimuth of 45 degrees and the 
analysis of the position given by the recreation grade receiver shows them to be $4.5 \mathrm{~m}$ apart at 43 degrees the error is $0.5 \mathrm{~m}$ and 2 degrees. In addition, in both case studies surveys were undertaken with devices being left for one and three minutes on each point before recording to assess the benefits of leaving a device to average repeated measurements.

The data collected from case study one and two were processed using the spatially enabled Oracle database and a specially developed python script respectively. The latter was developed on the basis of the first case study to establish a more readily available form of data processing. Python is not only more readily available to researchers and students, being open source, but can also be used in programmes such as ArcGIS. The specific python script developed for this method has been made readily available to readers on the online code repository GitHub (https://github.com/gisportsmouth/pythonforgis.git).

\section{Case Study 1 - Sinah Gun Site, Hayling Island, Hampshire, UK}

The site chosen for case study one comprised twin octagonal concrete structures ca. $14 \mathrm{~m}$ across and ca. 20 metres apart on Hayling Island, Hampshire, UK (Figure 2). These structures were chosen for their regular geometric shape which can highlight the errors in relative accuracy. In addition, the site provided a clear view of the sky, limiting the impact on the receiver bias through multi-path error. 
Figure 2: Orthophoto of Sinah Gun site (Channel Coast 2011).

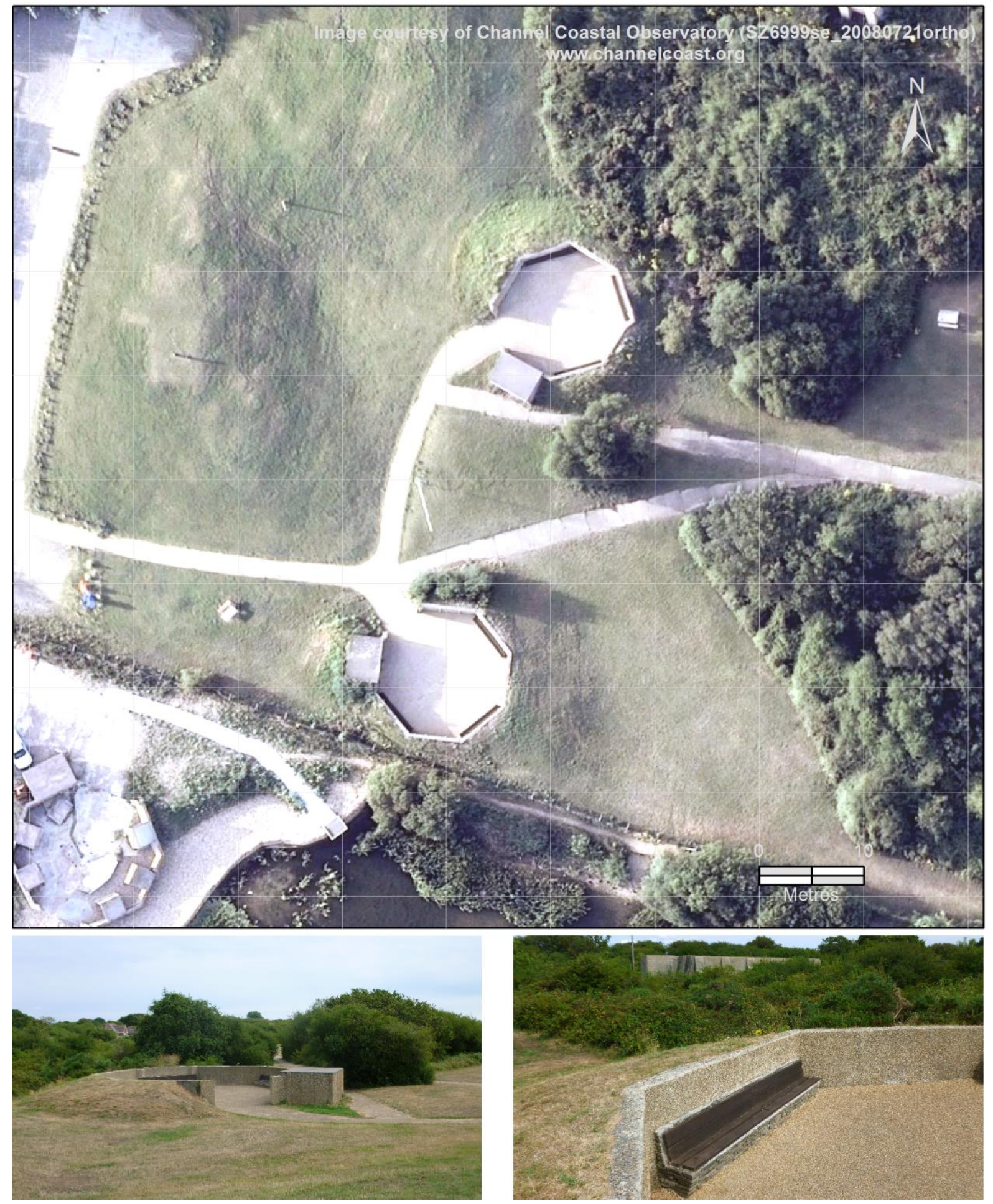

A base was established using a local Ordnance Survey (OS) passive station and a Topcon HiperPro DGNSS. OS passive stations have "ETRS89 coordinates with (typically) $5 \mathrm{~cm}$ horizontal accuracy (better for some stations)" (OS 2010 n.p.). The survey site was surveyed 
using a Trimble 5600 TS. The positional accuracy of the survey can be assumed to be $5 \mathrm{~cm}$ and the relative accuracy $1 \mathrm{~cm}$. Where applicable, consistent, high-accuracy geographic transformations were used. The results can be seen in Figure 3.

Figure 3: Map of accurate Sinah Gun site survey data.

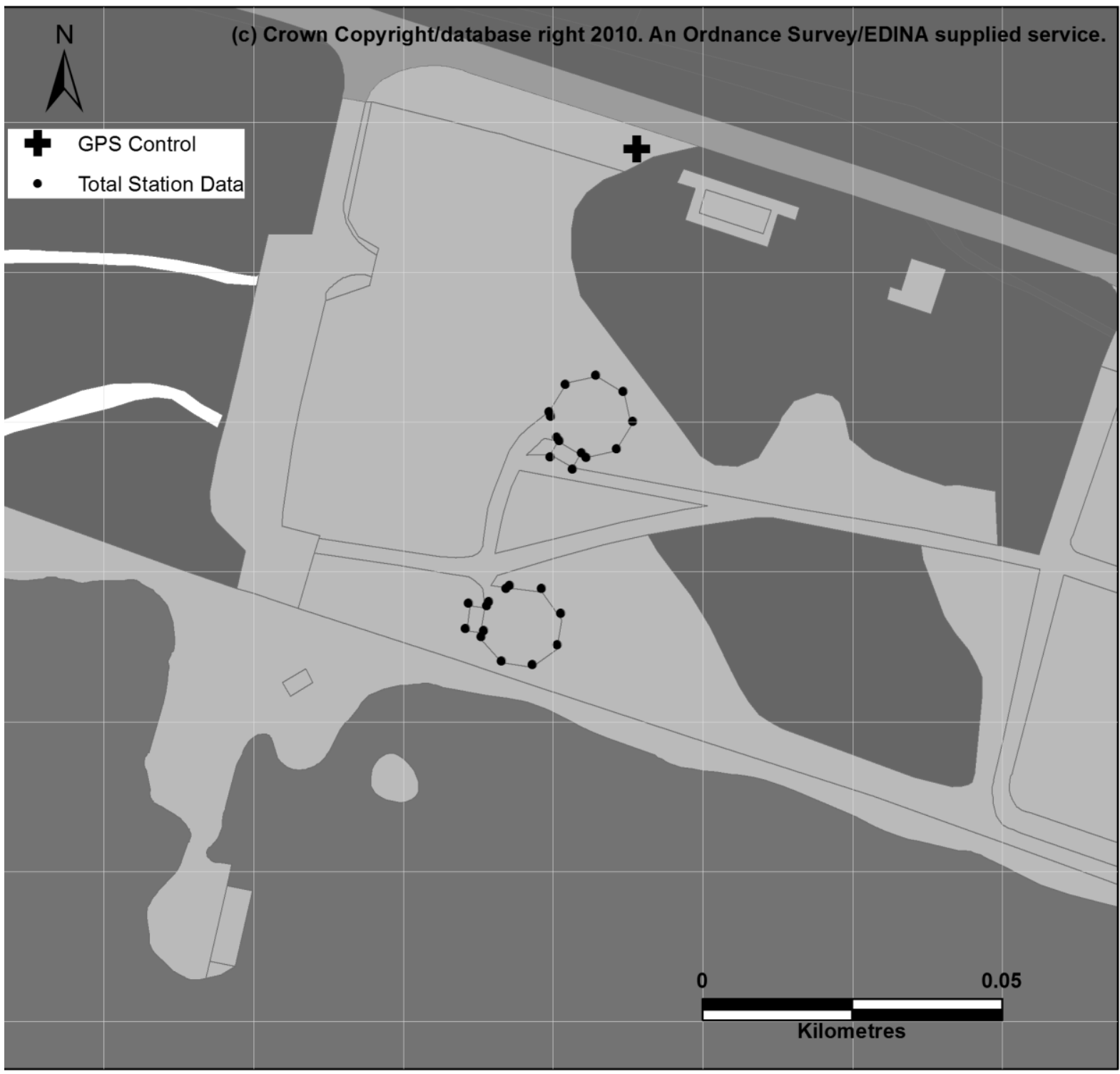

The recreation grade receivers were used to collect data over several surveys on different days. The recreation grade devices used were:

- 2 Garmin eTrex recreation grade GPS (Garmin) Software version 3.6,

- 2 Magellan SporTrak recreation grade GPS (Magellan) rev 1.1 2002, 
For each survey, defined as recording all points of the hexagonal features without interruption with the same device, the unit ID and brand were recorded. In addition to the position and elevation recorded by the unit, a number of factors were recorded manually for each reading:

- The number of satellites being tracked

- The stated accuracy by the unit

- The time and date

- The presence or absence of a Wide Area Augmentation System (WAAS)

In the Garmin Units it was possible to switch off WAAS, which was done for two surveys to examine any influence on levels of accuracy.

There were six surveys using the Magellan GPS and five using the Garmin GPS, providing 285 statistical data points for absolute accuracy assessment and 3550 for relative accuracy. The display of the recreation grade units give degrees and decimal minutes to 3 places for each point, the output of the recreation grade units when downloaded provides degrees Latitude and Longitude in WGS84 to 6 decimal places, implying that the precision of the data is to 11 centimetres (with reference to Latitude at the Equator based on WGS84 Ellipsoid). As the precision of the output is in the decimetre range all distance values were computed in metres to a precision of one decimal place.

The horizontal deviation from the base data, the absolute accuracy, showed that the mean deviation from the base positions is $2.1 \mathrm{~m}$, with a standard deviation of 1.11 (see Table II). 
Table II: Descriptive statistics of recreation grade GNSS device accuracy, Sinah Gun site

\begin{tabular}{|l|r|r|r|r|r|r|}
\hline $\begin{array}{l}\text { Measure of recreation grade GPS receivers (all } \\
\text { values in Metres) }\end{array}$ & $\mathrm{N}$ & Mean & $\begin{array}{c}\text { Std. } \\
\text { Deviation }\end{array}$ & Range & Minimum & Maximum \\
\hline Horizontal absolute accuracy & 285 & 2.07 & 1.11 & 7.4 & 0.1 & 7.5 \\
\hline $\begin{array}{l}\text { Vertical absolute accuracy of recreation grade GPS } \\
\text { (RMSE) }\end{array}$ & 285 & 2.01 & 1.72 & 10 & 0 & 10 \\
\hline $\begin{array}{l}\text { Horizontal relative accuracy of recreation grade } \\
\text { GPS receivers within the same survey (RMSE) }\end{array}$ & 3550 & 1.42 & 1.11 & 7.89 & 0 & 7.89 \\
\hline $\begin{array}{l}\text { Vertical relative accuracy of recreation grade GPS } \\
\text { receivers within the same survey (RMSE) }\end{array}$ & 3550 & 2.81 & 2.24 & 15.08 & 0 & 15.08 \\
\hline
\end{tabular}

The perceived external predictors of horizontal accuracy were the brand of device, the time left for the device to settle (one or three minutes; also referred to as observation time) and if WAAS was present (see Table III). These factors were considered in a regression model along with the survey instance, the number of satellites and the stated accuracy of the device. A stepwise regression showed that only the stated accuracy had a significant correlation with the horizontal deviation.

Table III: Independent sample t-Tests results for external factors, Sinah Gun Site

\begin{tabular}{|l|l|c|c|}
\hline Response & Predictor & $\begin{array}{l}\text { Equal } \\
\text { Variance? }\end{array}$ & $\begin{array}{l}\text { Equal } \\
\text { Mean? }\end{array}$ \\
\hline $\begin{array}{l}\text { Horizontal absolute } \\
\text { accuracy }\end{array}$ & by Brand & Yes & Yes \\
\hline $\begin{array}{l}\text { Horizontal absolute } \\
\text { accuracy }\end{array}$ & $\begin{array}{l}\text { by Observation } \\
\text { time }\left(1^{\prime} / 3^{\prime}\right)\end{array}$ & No & Yes \\
\hline $\begin{array}{l}\text { Horizontal absolute } \\
\text { accuracy }\end{array}$ & $\begin{array}{l}\text { by WAAS } \\
\text { (On/Off) }\end{array}$ & Yes & Yes \\
\hline Vertical absolute accuracy & by Brand & No & No \\
\hline Horizontal relative accuracy & by Brand & No & No \\
\hline Horizontal relative accuracy & $\begin{array}{l}\text { by Observation } \\
\text { time (1'/3') }\end{array}$ & No & No \\
\hline
\end{tabular}

The vertical accuracy for high precision survey GNSS receivers is often described as 1.5 to 2 times less accurate than the horizontal precision (Topcon 2005; Trimble 2008). The base data set has a limited height range; the range is only $0.72 \mathrm{~m}$. The root mean square error (RMS) 
shows an average of $2.01 \mathrm{~m}$ and a standard deviation of 1.72 (Table II). When comparing the two recreation grade receiver brands there is a significant difference between the means and the variances cannot be assumed to be equal (see Table III).

In terms of absolute accuracy, then, there is no significant difference in horizontal accuracy between brands of device; however, differences in vertical accuracy require consideration.

The resulting RMS error for the relative accuracy results shows a mean distance error of $1.42 \mathrm{~m}$ and a standard deviation of 1.11 (see Table II), compared to the absolute accuracy mean distance error of $2.07 \mathrm{~m}$.

The derived azimuth error data was normalised, i.e. converted into positives and data above 180 was subtracted from 360 to gain angular deviation, rather than azimuth. The azimuth error is significantly affected by the distance two points are apart. Quite ambitiously some of the closest points in the survey are only $0.7 \mathrm{~m}, 0.8 \mathrm{~m}$ and $1.1 \mathrm{~m}$ apart, respectively. Over a smaller distance any deviation will naturally produce a larger azimuth error. In addition points can overlap, producing the very high values in azimuth error (see Figure 4). Figure 5 and 6 show examples of how the recreation grade receiver created shapes compare to the control shapes. It can be seen that the major problems lie in the rectangular outcrops of the features where points are close together. 
Figure 4: Plot of azimuth error and horizontal distance of points, Sinah Gun site.

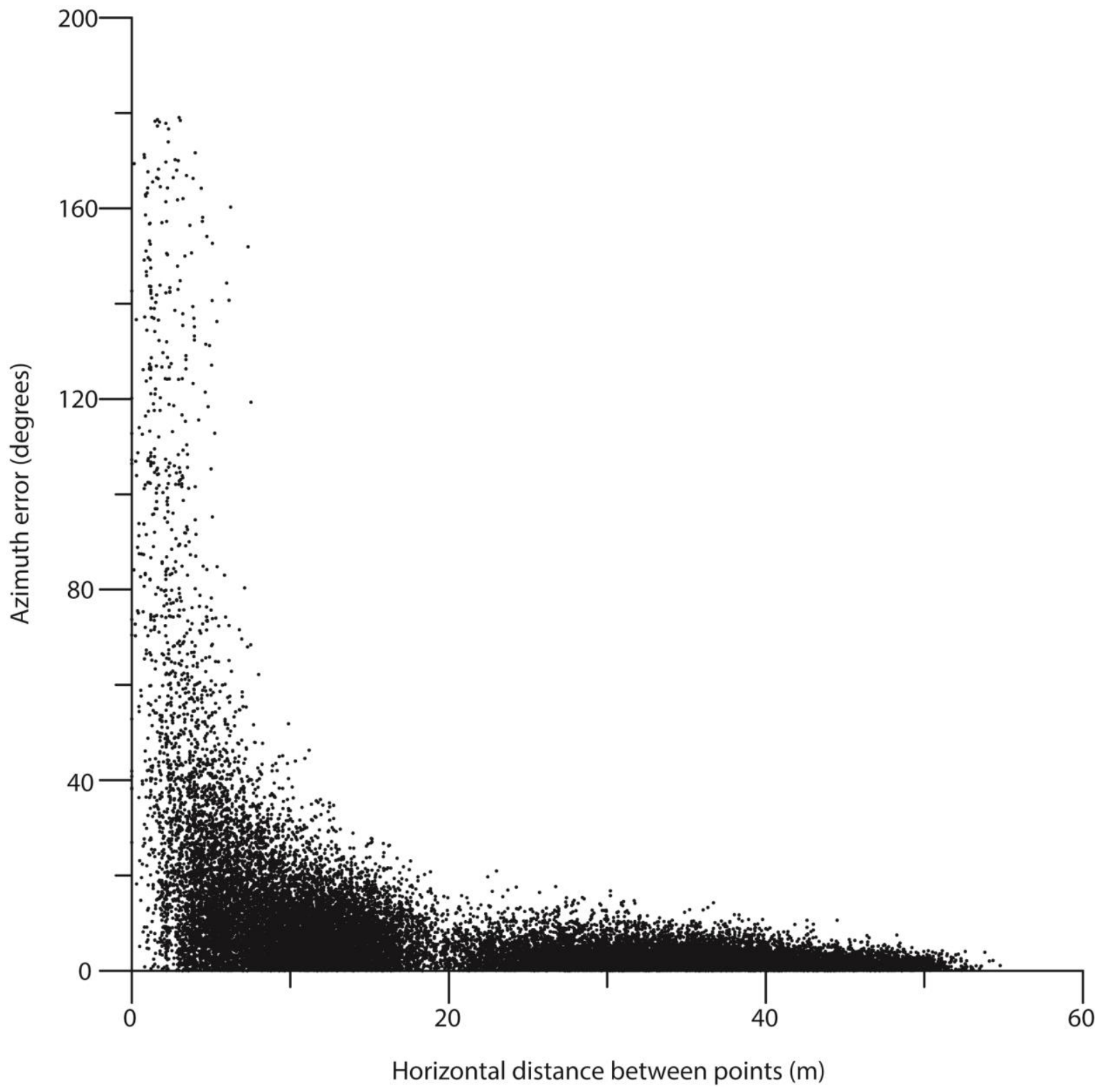


Figure 5: Comparison of control polygon shape and selected Garmin eTrex surveys, Sinah Gun Site
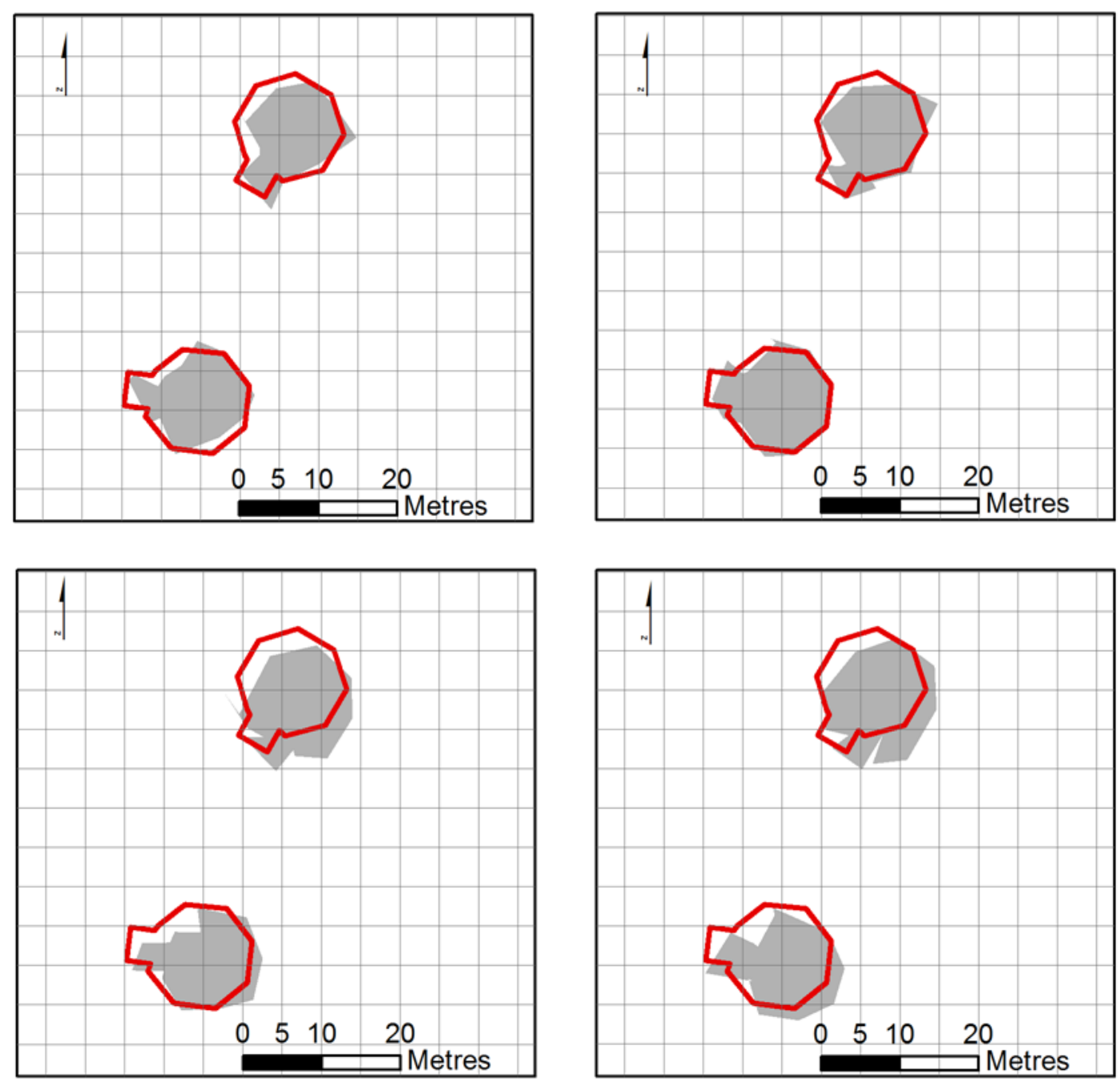

$\square$ Control Survey Garmin 
Figure 6: Comparison of control polygon shape and selected Magellan SporTrak surveys,

Sinah Gun site
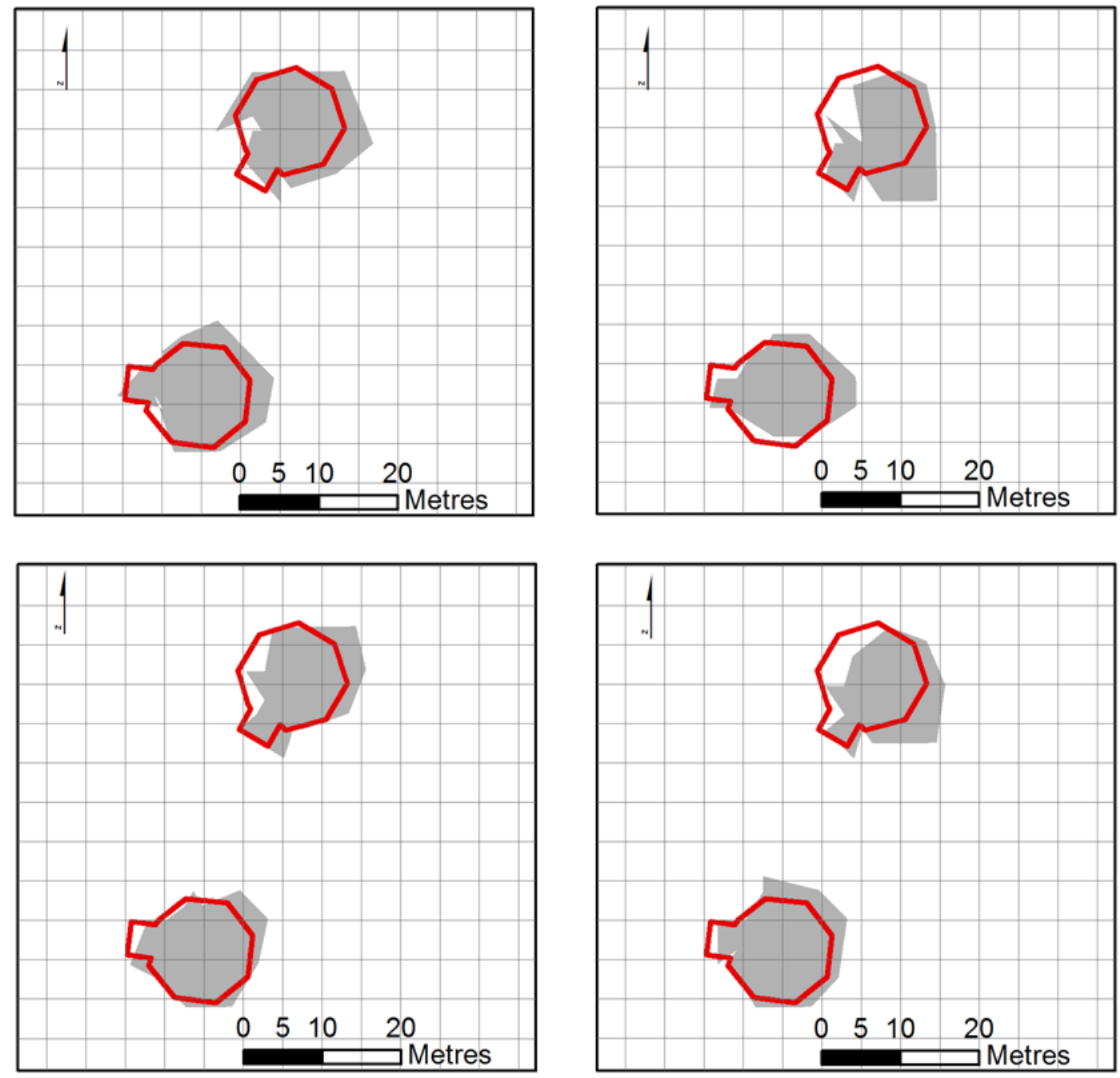

Control Survey Magellan

The relative accuracy was assessed according to the brand of the receiver and observation time, as summarised in Table II. The recreation grade receivers were found to have significantly different means, the Magellan having a mean of $1.66 \mathrm{~m}$ compared to the Garmin's $1.15 \mathrm{~m}$, and standard deviations of 1.24 for the Magellan and 0.87 for the Garmin. In order to explain the variance in the distance error the same factors were considered in a regression model as with the absolute accuracy assessment. In addition, the time between the survey readings was introduced as this determines difference in systematic error. 
Although the model is significant the r-squared of the model is low and the observation time only has a small positive effect on the model $(0.09 \mathrm{~m})$. The stated accuracy is again correlated with the error. There is a significant and noticeable effect of the brand of receiver $(0.5 \mathrm{~m})$ and a significant negative effect of time between readings $(0.18 \mathrm{~m} /$ hour $)$. The intra survey results indicate that relative accuracy is, therefore, improved with a longer settling time for the device, and a shorter period between readings.

\section{Case Study 2 - Southsea Promenade, Southsea, Hampshire, UK}

The site chosen for case study two was the easily identifiable markings on the ground on Southsea Promenade in Southsea, Hampshire, UK (Figure 7). Twenty points were surveyed using a Topcon HiPer Pro DGNSS, with a base established using the OS active station network. Accuracy is assumed to be within $5 \mathrm{~cm}$ based on OS specifications. Student volunteers were instructed to survey the points using their mobile phone devices (listed in Table I), one survey for 1 minute and a second survey for 3 minutes each; elevation was not considered in this case study. As with crowdsourced data in general, there can be an increased amount of noise to the data and additional scrutiny is required, but data can be good nonetheless (Heipke 2010; Hsueh et al 2009). Some initial validation to remove obvious outliers was performed and a small number of data sets were excluded before analysis. 
Figure 7: Orthophoto of Southsea study site (Channel Coast 2011).

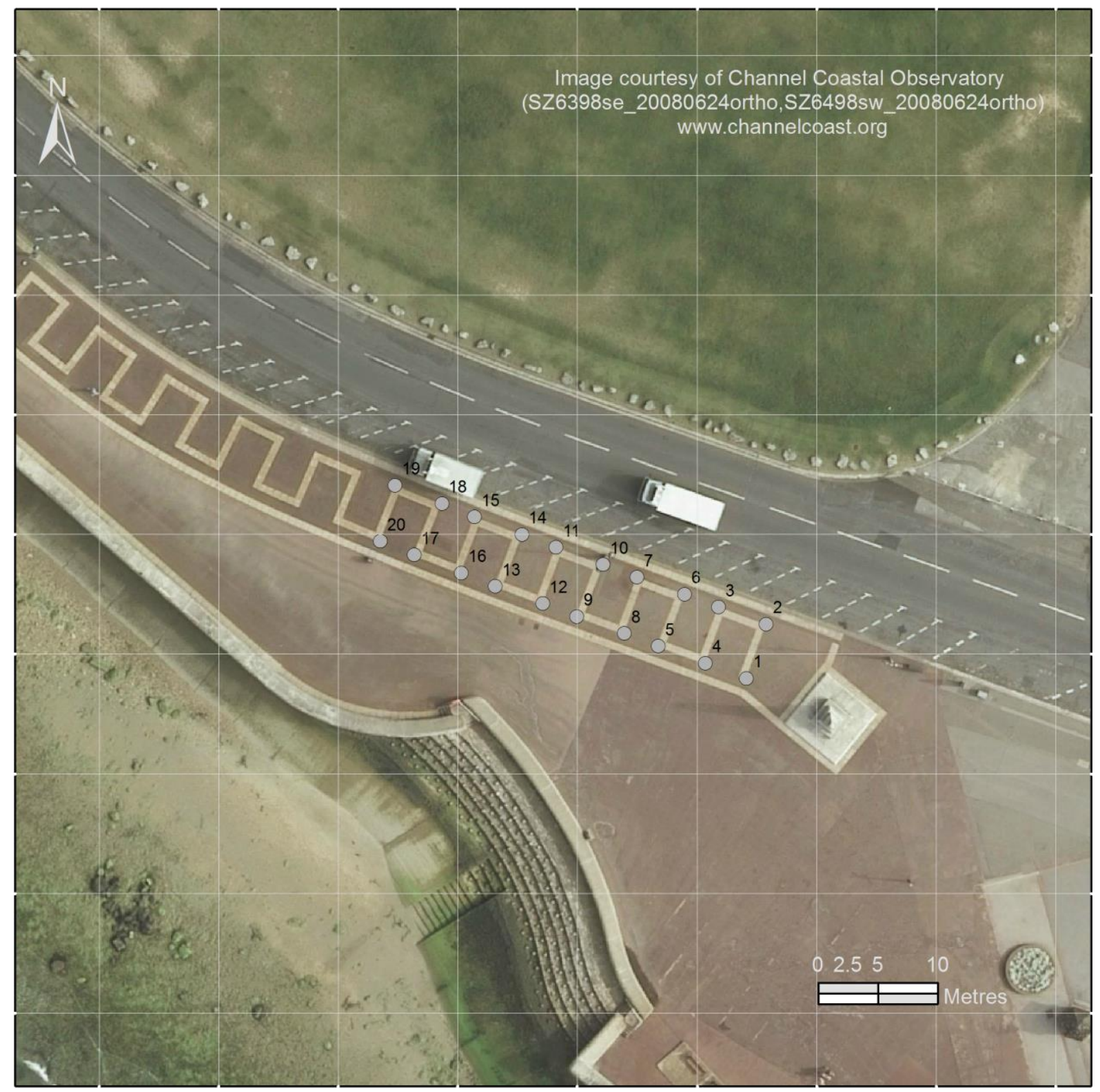

On the Apple devices position was recorded manually, as no free point collection application to fit the purposes of the study could be found. On Android an application called Locus Free by Asamm Software was used that easily outputs in GPS Exchange Format (GPX). In all cases the coordinate reference system used was WGS84. 
A python script was created to quickly process both accuracies and to enable the students to see the results immediately post survey. The script outputs tables of individual data for each point, as well as summary files of both accuracies.

Of the devices used (Table III) all the Apple and Samsung phones use GPS and GLONASS, as does the Sony Xperia Z. The Xperia E and P do not use GLONASS (GSMArena.com 2014). There were 13 surveys using Apple phones, 6 using Samsung phones and 8 using Sony Xperia models, providing 558 statistical data points for absolute accuracy assessment and 6026 for relative accuracy. Output is in latitude and longitude to 6 decimal places in all cases.

The horizontal deviation from the base data, the absolute accuracy, showed that the mean deviation from the base positions is $3.49 \mathrm{~m}$, with a standard deviation of 3.67 (see Table IV). The range is 44.07 .

Table IV: Descriptive statistics of mobile phone GNSS data, Southsea survey site

\begin{tabular}{|c|c|c|c|c|c|c|}
\hline $\begin{array}{l}\text { Measure of mobile phone GPS receivers (all values in } \\
\text { metres) }\end{array}$ & $\mathrm{N}$ & Mean & $\begin{array}{c}\text { Std. } \\
\text { Deviation }\end{array}$ & Range & Minimum & Maximum \\
\hline Horizontal absolute accuracy (combined) & 638 & 3.49 & 3.67 & 43.96 & 0.1 & 44.07 \\
\hline Horizontal absolute accuracy (Apple iPhone 4) & 40 & 2.65 & 1.27 & 5.37 & .78 & 6.15 \\
\hline Horizontal absolute accuracy (Apple iPhone 5) & 300 & 4.19 & 4.71 & 43.82 & .26 & 44.07 \\
\hline Horizontal absolute accuracy (Samsung) & 120 & 2.75 & 1.69 & 10.39 & .25 & 10.65 \\
\hline Horizontal absolute accuracy (Sony) & 158 & 2.64 & 2.06 & 17.65 & .11 & 17.76 \\
\hline Horizontal relative accuracy RMSE (combined) & 6026 & 2.44 & 3.65 & 45.23 & 0 & 45.23 \\
\hline Horizontal relative accuracy RMSE (Apple iPhone 4) & 380 & 1.67 & 1.31 & 7.23 & .01 & 7.24 \\
\hline Horizontal relative accuracy RMSE (Apple iPhone 5) & 2850 & 3.03 & 4.78 & 45.23 & 0 & 45.23 \\
\hline Horizontal relative accuracy RMSE (Samsung) & 1140 & 1.85 & 1.70 & 10.29 & 0 & 10.29 \\
\hline Horizontal relative accuracy RMSE (Sony) & 1466 & 1.71 & 2.87 & 14.68 & 0 & 14.68 \\
\hline
\end{tabular}


There is a significant difference in mean and standard deviation between the iPhone 5 on one hand and the iPhone 4, Samsung and Sony devices on the other. There is no difference between iPhone 4, Samsung and Sony devices.

There is no significant difference between data collected with GLONASS enabled and nonenabled Sony devices. There is also no significant difference between the different occupation times; waiting for 3 minutes instead of 1 minute showed no difference. Specialist applications that use an averaging function were not considered in this case study.

The resulting RMS error for the relative accuracy results shows a mean distance error of $2.44 \mathrm{~m}$ and a standard deviation of 3.65 (see Table IV), compared to the absolute accuracy mean distance error of $3.49 \mathrm{~m}$. The range is 45.23 .As above, the difference between iPhone 5 and iPhone 4, Samsung and Sony devices is significant.

There is a significant difference between the mean of data collected with GLONASS enabled $(1.99 \mathrm{~m})$ and non-enabled Sony devices $(1.45 \mathrm{~m})$, with the non-GLONASS devices showing a better relative accuracy and less variation (1.23 compared to 2.04).

The occupation time of 1 minute is significantly better $(2.1 \mathrm{~m})$ than 3 minutes $(2.78 \mathrm{~m})$ with significantly different variance (3.16 compared to 4.05$)$. 


\section{Discussion}

In the case of recreation grade receivers the absolute accuracy was found to follow, if not exceed, manufacturer's specifications and the stated accuracy of the device. The analysis of the absolute accuracy showed that $95 \%$ of readings can be expected to be within $4.29 \mathrm{~m}$ of the correct horizontal location, with a mean of $2.07 \mathrm{~m}$. The stated accuracy of the devices in the device display correlated with the absolute horizontal accuracy, demonstrating that they are a good indicator of GNSS accuracy. The absolute vertical measurements of the recreation grade receivers were found to be less accurate and more variable than the horizontal accuracy. The analysis shows that while increasing the observation time of the receivers does not affect the mean error, it does reduce the variability of the results. The presence of WAAS did not show as a significant factor in this study. Caution has to be shown when features are closer together than the absolute accuracy if the shape of features is to be surveyed. The analysis shows that the azimuth error is more pronounced if two points are close together. Over larger distances the resulting distance between feature values and the shape of connecting features can be assumed to be better than the absolute accuracy given by the device.

This study indicates that in practical terms either of the 12-channel receivers can be employed and it is better to leave the device to settle. It should, however, be noted that whilst the brand of recreation grade receiver was not found to influence the absolute horizontal accuracy within this study, this is in contrast to the findings of Wing et al. (2005). In a study testing the accuracy and reliability of recreation grade receivers in a variety of landscape settings (open sky, young forest and closed canopy), they found that accuracy between brands varied, "in some cases considerably" (p.171). Accuracy under open sky conditions was found to range from $2.6 \mathrm{~m}$ (mean 1.4) to $27.2 \mathrm{~m}$ (mean 19.6), however, they note the anomalous performance 
of two particular devices from their sample of six, and limitations in the software used in their study. Wing et al. (2005) found that the top four recreation grade models were found to be "within approximately 5m of true position" (p.172). They also used Garmin and Magellan receivers although the models did not exactly match this study's models.

The mobile phones displayed more varied results. In general they do not perform as well as the recreation grade devices in terms of absolute accuracy; they, in particular, were more variable with bigger ranges. Although some showed good results, variation was found between models of the same brand.

In terms of relative accuracy, a better accuracy can be expected, especially if the total survey is conducted over a short time window, and this can inform the choice of survey strategy. The analysis shows that for recreation grade devices $95 \%$ of readings can be expected to be within $3.63 \mathrm{~m}$ with a mean of 1.41 within the same survey, although the time between readings was found to have a significant impact, with deviation increasing with time. Mobile phones also improved when looking at relative accuracy; they did not, however, show the same decrease in variability over longer observation times.

The positional readings in both studies were taken in ideal conditions regarding sky view. A research setting might not be as forgiving, with tree canopies or tall buildings having a major influence on accuracy. For example, Wing (2009) found that the most accurate recreation grade receiver in their sample of six different devices had average measurement errors of approximately $2 \mathrm{~m}$ or less in open sky conditions, $3 \mathrm{~m}$ or less in a young forest setting, and 9 m or less under a closed canopy. Similarly, Rodríguez-Pérez et al. (2007) calculated accuracy errors in excess of $5 \mathrm{~m}$ in their assessment of recreation grade receivers in forested areas. 
This analysis demonstrates that recreation grade receivers and mobile phones offer potential users a relatively inexpensive and manageable technology for collecting coordinate locations of landscape features, particularly in open sky settings and with regards to relative accuracy. Given the advance of GNSS technology (for example, improvements in SBAS and new systems) it has been shown that it is important to have an appreciation of any proposed device's accuracy. Setting up an accurate benchmark that can be revisited by students and researchers offers a good approach, coupled with the ease of running a python script once the base has been established. In that way each new device can be assessed and a comparative dataset gained over time for further analysis and dissemination. The results also present a 'best practice' approach to recreation grade devices in a research and study context. A single device should be used over a short time period ( $<4$ hours in the case of this study) and left to settle before recording a point. Mobile phones might benefit from a specialist averaging application, data from which could be tested following the above system.

\section{Conclusions}

Notwithstanding the present study, there remains limited empirical evidence of the relative accuracy of low-cost GNSS receivers in open areas. Given the levels of relative accuracy demonstrated, the present study shows that this is a subject deserving more attention. Existing studies of accuracy have largely concentrated on absolute accuracy, e.g. in forestry studies on how different forest conditions, particularly canopy cover, can affect levels of accuracy (Andersen et al. 2009; Rodríguez-Pérez et al. 2007; Wing 2009; Wing et al. 2005).

The levels of relative accuracy displayed by recreation grade receivers and mobile phone devices, combined with their affordability/availability and portability in comparison to 
commercial survey DGNSS, make these devices worthy of consideration for student fieldwork and research. The method presented in this paper offers a useful means of evaluating the levels of absolute and relative accuracy, which can inform the choice of device for particular survey-based projects. With the advancement of both GNSS and mobile technologies the continuing evaluation of these devices, and the potentially new areas of use given increased accuracy performance, will be an ongoing requirement for the GNSS professional supporting end users of the technology. Raising awareness of relative accuracy will be an important part of this support.

Using this method of evaluation can also be a useful exercise in and of itself for helping students better understand absolute and relative accuracy, and the potential use of recreation grade and mobile phone devices in a range of scenarios both within and beyond the academy, and gauge more fully the capabilities of their own mobile hardware.

\section{Acknowledgements}

Thanks go to Sophie Bennett for organising and collating the mobile phone survey, and Dr. Liz Twigg and Dr. Rob Inkpen for constructive advice on paper content. 


\section{References}

Andersen H E, Clarkin T, Winterberger K and Strunk J 2009 An accuracy assessment of positions obtained using survey- and recreational- grade global positioning system receivers across a range of forest conditions within the Tanana valley of interior Alaska. Western Journal of Applied Forestry 24(3) 128-136

Al-Husain L, Kanjo E and Chamberlain A 2013 Sense of Space: mapping physiological emotion response in urban space Proceedings of the 2013 ACM conference on Pervasive and ubiquitous computing adjunct publication Association for Computing Machinery 1321-1324

Channel Coast Observatory 2011 Othophoto of study site SZ6999se_200080721 ortho. Downloaded from channelcoast.org 02/07/2011

European GNSS Agency 2013 Galileo Is The European Global Satellite-Based Navigation System ( http://www.gsa.europa.eu/galileo-0) Accessed 29 October 2013

Franchois A 2005 Determination of GPS positioning errors due to multi-path in civil aviation Proceedings of 2nd International Conference on Recent Advances in Space Technologies. RAST 2005. Proceedings of 2nd International Conference 2005: 400-403

Gleason S, Gebre-Egziabher, D 2009 GNSS Applications and Methods Artech House, Boston, Mass

Heipke C 2010. Crowdsourcing geospatial data. ISPRS Journal of Photogrammetry and Remote Sensing, 65(6), 550-557

Heßelbarth A and Wanninger L 2012 SBAS orbit and satellite corrections for precise point positioning. GPS Solutions 17 465-473

Hsueh P, Melville P and Sindhwani V 2009 Data quality from crowdsourcing: a study of annotation selection criteria Proceedings of the NAACL HLT 2009 workshop on active learning for natural language processing, 27-35 
Jyh-Ching J, Yung-Fu T and Chung-Huei C 2013 On constellation design of multi-GNSS radio occultation mission. Acta Astronautica 82 88-94

Kaplan ED and Hegarty CJ 2006 Understanding GPS: principles and applications Artech House Publishers, Boston, Mass

Longley P, Goodchild MF, Maguire D and Rhind D 2011 Geographic information systems and science, $3^{\text {rd }}$ edition Wiley, New York

O'Shea, D 2013 Real-World Drive Tests Declare A Verdict On GPS/GLONASS (http://electronicdesign.com/test-amp-measurement/real-world-drive-tests-declare-verdictgpsglonass) Accessed 17 November 2014

Ordnance Survey 2012 GNSS and positioning services FAQs [online] (http://www.ordnancesurvey.co.uk/oswebsite/gps/osnetfreeservices/about/faqs_osnet.html) Accessed 3 November 2012

Ordnance Survey 2013 Active Station RINEX Data[online] (http://gps.ordnancesurvey.co.uk/active.asp) Accessed 25 January 2013

OXTS 2014 What does GLONASS add? (http://www.oxts.com/technical-notes/what-doesglonass-add/) Accessed 17 November 2014

Rodríguez-Pérez JR, Álvarez MF and Sanz-Ablanedo E 2007 Assessment of Low-Cost GPS Receiver Accuracy and Precision in Forest Environments. Journal of Surveying Engineering November 159-167

Sigrist P, Coppin P and Hermy M 1999 Impact of forest canopy on quality and accuracy of GPS measurements International Journal of Remote Sensing 20(18) 3595 -3610

Trimble 2008 Trimble 5800 GPS System Data Sheet. Topcon 2005 HiPer Pro GSP + Receivers Leaflet

Townsend B and Fenton P 1994 A practical approach to the reduction of pseudorange multipath errors in a L1 GPS receiver. Proceedings of ION GPS-94, 143-148 
Uren J, Price WF 2006 Surveying for engineers, $4^{\text {th }}$ edition Palgrave Macmillan, Basingstoke

Wing M 2009 Consumer-Grade Global Positioning Systems (GPS) Receiver Performance Journal of Forestry 106(4) 185-190

Wing M G, Eklund A and Kellogg LD 2005 Consumer-grade global positioning system (GPS) accuracy and reliability Journal of Forestry 103(4) 169-173

Van Sickle J 2008 GPS for land surveyors, $3^{\text {rd }}$ edition CRC, Boca Raton, Fla 